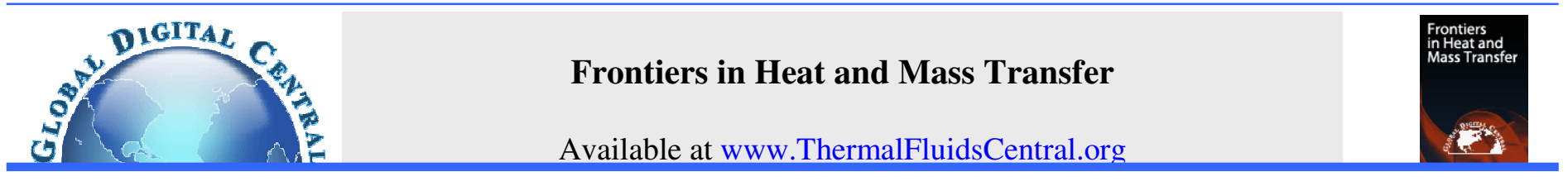

\title{
ANALYTICAL INVESTIGATIONS OF DIFFUSION THERMO EFFECTS ON UNSTEADY FREE CONVECTION FLOW PAST AN ACCELERATED VERTICAL PLATE
}

\author{
E. Kumaresan ${ }^{a}$, A .G. Vijaya Kumar ${ }^{a *}$ and J. Prakash ${ }^{\mathrm{b}}$ \\ ${ }^{a}$ Department of Mathematics, School of Advanced Sciences, VIT University, Vellore, TN, 632 014, India \\ ${ }^{b}$ Department of Mathematics, University of Botswana, Private Bag 0022, Gaborone, BOTSWANA
}

\begin{abstract}
The objective of this study is to investigate diffusion-thermo and radiation effects on unsteady free convection chemically reacting fluid flow past an accelerated infinite plate with variable temperature and mass diffusion under the influence of uniform transverse magnetic field when the magnetic lines of force are fixed relative to the fluid or to the plate. . Two important cases, when the magnetic lines of force are being fixed relative to the fluid $(K=0)$ or to the moving plate $(K=1)$ have been considered. A general exact solution of the dimensionless governing partial differential equations is obtained by Laplace transform technique without any restriction. The effects of various parameters describing the flow transport in the presence of thermal radiation and Dufour effect on the fluid velocity, temperature, concentration, the rate of heat transfer and the rate of mass transfer are concentrated on through graphs.

Keywords: $M H D$, Dufour effect, radiation, free convection, exponentially accelerated plate, chemical reaction.
\end{abstract}

\section{INTRODUCTION}

In nature, there exist flows which are caused not only by the temperature differences but also the concentration differences. These mass transfer differences do affect the rate of heat transfer. In industries, many transport processes exist in which heat and mass transfer takes place simultaneously as a result of combined buoyancy effect in the presence of thermal radiation. Hence, radiative heat and mass transfer play an important role in manufacturing industries for the design of fins, steel rolling, nuclear power plants, gas turbines and various propulsion device for aircraft, missiles, satellites, combustion and furnace design, materials processing, energy utilization, temperature measurements, remote sensing for astronomy and space exploration, food processing and cryogenic engineering, as well as numerous agricultural, health and military applications. If the temperature of surrounding fluid is rather high, radiation effects play an important role and this situation does exist in space technology. In such cases, one has to take into account the combined effect of thermal radiation and mass diffusion.

The Effects of thermal diffusion and radiation on unsteady MHD free convection flow past an infinite heated vertical plate in a porous medium was studied by Sivaiah et al. (2012). Hetnarstki (1975) and Puri and Kythe (1988) reported an algorithm for generating some inverse transforms of exponential form. Narahari and Debnath (2013) investigated unsteady magnetohydrodynamic free convection flow past an accelerated vertical plate with constant heat flux and heat generation or absorption by Laplace Transform method. Prabhakar Reddy (2014) studied effects of thermal diffusion and viscous dissipation on unsteady MHD free convection flow past a vertical porous plate under oscillatory suction velocity with the heat sink. Seth et al. (2010) investigated MHD natural convection flow past an impulsively moving vertical plate with ramped wall temperature in the presence of thermal diffusion with heat absorption. The effects of thermal radiation and heat source on an unsteady MHD free convection flow past an infinite vertical plate with thermal diffusion and diffusion thermo was analyzed by Raju et al. (2013). Prakash et al. (2013) examined the effects of diffusion-thermo and radiation on unsteady MHD free convective flow with variable temperature and mass diffusion. In all the above studies, the stationary vertical plate is considered. Muthucumaraswamy et al. (2004) and Rajesh et al. (2009) studied radiation and mass transfer effects on exponentially accelerated isothermal vertical plate. Muralidharan and Muthucumaraswamy (2013) analyzed Radiation effects on linearly accelerated isothermal vertical plate with variable mass diffusion in the presence of magnetic field. Rushi Kumar et al. (2015) investigated the influence of magnetic field in the presence of thermal radiation and diffusion on a past vertical plate when magnetic lines relative to the fluid or the plate.

An energy flux is generated not only by temperature gradients but by composition gradients as well. Temperature gradients can also create mass fluxes and this is the Soret or thermal-diffusion effect. Generally, the thermo-diffusion and diffusion-thermo effects of smaller order magnitude than the effects prescribed by Fourier's or Fick's laws and are often neglected in heat and mass transfer processes. Due to the importance of thermo-diffusion and diffusion-thermo effects for the fluids with very light molecular weight as well as medium molecular weight, many investigators have studied and reported results for these flows and the contributors such as Eckert and Drake (1972), Anghel et al.(1985), Postelnicu (2004) are worth mentioning. Mythreye et al. (2015) analyzed the chemical reaction on unsteady MHD convective heat and mass transfer past a semi infinite vertical permeable moving plate with heat absorption. Venkateswarlu et al. (2014) analyzed thermal diffusion and radiation effects on unsteady MHD free convection heat and mass transfer flow past a linearly accelerated vertical porous plate with variable temperature and mass diffusion. Alam et al. [(2005), (2006), (2006a), and (2006b)] investigated the Dufour and Soret effects on unsteady laminar viscous 
incompressible MHD free convection and mass transfer flow past an impulsively started infinite vertical plate embedded in a porous medium under the influence of transverse applied a magnetic field.

Georgantopoloulos et al. (1979) studied the magnetohydrodynamic free convection flow past an impulsively started infinite vertical plate with uniform temperature. Ogulu and Makinde (2009) investigated unsteady hydromagnetic free convection flow of a dissipative and rotating fluid past a vertical plate with constant heat flux. Toki and Tokis (2007) investigated exact solutions for the unsteady free convection flows on a porous plate with time-dependent heating. Swetha et al. (2015) studied the effects of thermal radiation and radiation absorption on flow past an impulsively started infinite vertical plate with Newtonian heating and chemical reaction. Several studies were continued on magneto-hydrodynamic free convection flows past a vertical surface under different boundary conditions for various physical situations. Chandran et al. (1998) was investigated the effects of magnetic field and buoyancy force on the unsteady free convection laminar flow of an electrically conducting fluid when the flow was generated by uniformly accelerated motion of an infinite inclined plate subjected to constant heat flux. They obtained an exact solution with the help of Laplace transform technique and the numerical results were computed with the approximated error functions appeared in the solution.

However, in the literature we found less attention was paid on unsteady MHD free convection flows past a vertical plate subjected to a variable temperature and uniform mass diffusion with Dufour effect when the magnetic lines being fixed relative to the fluid or to the moving plate even though this situation involves in many engineering applications such as in aeronautics, spacecraft design and analysis of thermal plumes into atmosphere which are responsible for atmospheric pollution. In this paper, it is proposed to study diffusion-thermo and radiation effects on free convection flow past an accelerated infinite vertical plate with variable temperature and mass diffusion discussed in two cases, when the magnetic lines of force are fixed relative to the fluid $(K=0)$ or to the plate $(K=1)$ in the presence of transverse applied a magnetic field and chemical reaction. The dimensionless governing equations are solved using Laplace transform technique and the solutions are expressed in terms of exponential and complementary error functions.

\section{MATHEMATICAL ANALYSIS}

Diffusion-thermo and radiation effects on unsteady MHD free convection of flow of a viscous incompressible, electrically conducting, radiating fluid past an impulsively started infinite plate with variable temperature and uniform mass diffusion in the presence of transverse applied magnetic field when the magnetic lines of force are fixed relative to the fluid or to the plate. Two important cases, when the magnetic lines of force are being fixed relative to the fluid $(K=0)$ or to the moving plate $(K=1)$ have been considered. The $x^{\prime}$-axis is taken along the plate in vertically upward direction and $y^{\prime}$-axis is taken normal to it in the direction of applied transverse magnetic field. Initially, it is assumed that the plate and surrounding fluid are at the same temperature and concentration in a stationary condition for all the points in the entire flow region $y^{\prime} \geq 0$. At the time $t^{\prime} \geq 0$, the plate is given an impulsive motion with constant velocity $u=u_{0} \exp \left(a_{0}^{\prime} t^{\prime}\right)$. At the same time, the plate temperature is raised linearly with time $t$ and the concentration levels near the plate are raised to $C_{w}^{\prime}$. A magnetic field of uniform strength $B_{0}$ is assumed to be applied normal to the flow. For free convection flow, it is also assumed that,

- $\quad$ The induced magnetic field is assumed to be negligible as the magnetic Reynolds number of the flow is taken to be very small.
- The viscous dissipation is neglected in the energy equation.

- The effects of variation in density $(\rho)$ with temperature and species concentration are considered only in the body force term, in accordance with usual Boussinesq approximation.

- The fluid considered here is gray, absorbing / emitting radiation but a non-scattering medium.

- Since the flow of the fluid is assumed to be in the direction of $x^{\prime}$ axis, so the physical quantities are functions of the space co-ordinate $y^{\prime}$ and $t^{\prime}$ only.

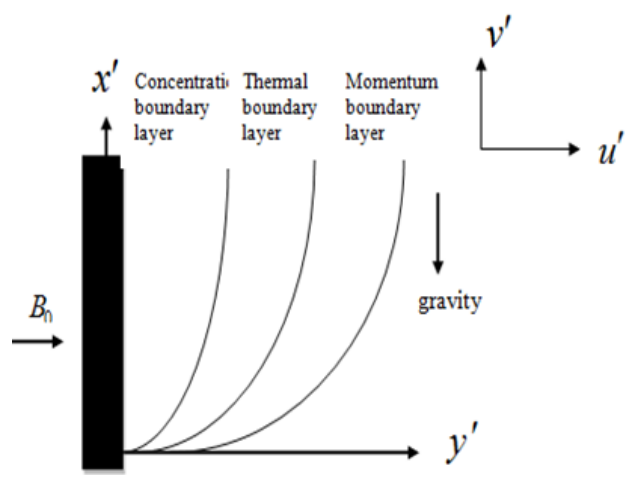

Fig. 1 Physical model

Then by usual Bossiness's approximation, the flow is governed by the following equations:

$$
\begin{aligned}
& \frac{\partial u^{\prime}}{\partial t^{\prime}}=g \beta\left(T^{\prime}-T_{\infty}^{\prime}\right)+g \beta^{*}\left(C^{\prime}-C_{\infty}^{\prime}\right)+v \frac{\partial^{2} u^{\prime}}{\partial y^{\prime 2}}-\frac{\sigma B_{0}^{2} u^{\prime}}{\rho} \\
& \rho c_{p} \frac{\partial T^{\prime}}{\partial t^{\prime}}=k \frac{\partial^{2} T^{\prime}}{\partial t^{\prime 2}}-\frac{\partial q_{r}}{\partial y}+\frac{D_{m} K_{T} \rho}{C_{s}} \frac{\partial^{2} C^{\prime}}{\partial y^{\prime 2}} \\
& \frac{\partial C^{\prime}}{\partial t^{\prime}}=D \frac{\partial^{2} C^{\prime}}{\partial y^{\prime 2}}-k^{\prime}\left(C^{\prime}-C_{\infty}^{\prime}\right)
\end{aligned}
$$

If the magnetic field fixed relative to the plate, the momentum equation (1) is replaced by (see [Raptis and Singh (1983), Tokis (1985), Cramer and Pai (1973)])

$$
\frac{\partial u^{\prime}}{\partial t^{\prime}}=g \beta\left(T^{\prime}-T_{\infty}^{\prime}\right)+g \beta^{*}\left(C^{\prime}-C_{\infty}^{\prime}\right)+v \frac{\partial^{2} u^{\prime}}{\partial y^{\prime 2}}-\frac{\sigma B_{0}^{2}}{\rho}\left[u^{\prime}-u_{0} f\left(t^{\prime}\right)\right]
$$

Note that the velocity $u_{0} f\left(t^{\prime}\right)$ of magnetic field $B_{0}$ in Eq. (4) appears because of the magnetic lines of force are fixed relative to the plate, which is accelerates with velocity $u_{0} f\left(t^{\prime}\right)$. Equation (1) and (4) can be combined as

$\frac{\partial u^{\prime}}{\partial t^{\prime}}=g \beta\left(T^{\prime}-T_{\infty}^{\prime}\right)+g \beta^{*}\left(C^{\prime}-C_{\infty}^{\prime}\right)+v \frac{\partial^{2} u^{\prime}}{\partial y^{\prime 2}}-\frac{\sigma B_{0}^{2}}{\rho}\left[u^{\prime}-K u_{0} f\left(t^{\prime}\right)\right]$

where $K=\left\{\begin{array}{l}0 \text { if } \mathrm{B}_{0} \text { is fixed relative to the fluid } \\ 1 \text { if } \mathrm{B}_{0} \text { is fixed relative to the plate }\end{array}\right.$

For an exponentially accelerated plate, $f\left(t^{\prime}\right)=\exp \left(a_{0}^{\prime} t^{\prime}\right)$, where $a_{0}^{\prime}$ is dimensional accelerating parameter.

With the following initial and boundary conditions

$$
\begin{aligned}
t^{\prime} \leq 0: u^{\prime} & =0, T^{\prime}=T_{\infty}^{\prime}, \quad C^{\prime}=C_{\infty}^{\prime}, \text { for all } y^{\prime} \\
t^{\prime}>0: u^{\prime} & =u_{0} \exp \left(a_{0}^{\prime} t^{\prime}\right), T^{\prime}=T_{\infty}^{\prime}+\left(T_{w}^{\prime}-T_{\infty}^{\prime}\right) A t^{\prime}, C^{\prime}=C_{w}^{\prime} \text { at } y^{\prime}=0 \\
& u^{\prime} \rightarrow 0, \quad T^{\prime} \rightarrow T_{\infty}^{\prime}, \quad C^{\prime} \rightarrow C_{\infty}^{\prime}, \quad \text { as } y^{\prime} \rightarrow \infty
\end{aligned}
$$


where $A=\frac{u_{0}^{2}}{v}$ the local radiant for the case of an optically thin gray gas is expressed by

$\frac{\partial q_{r}}{\partial y^{\prime}}=-4 a^{*} \sigma\left(T_{\infty}^{\prime 4}-T^{\prime 4}\right)$

It is assumed that the temperature differences within the flow are sufficiently small and that $T^{\prime 4}$ may be expressed as a linear function of the temperature. This is obtained by expanding $T^{\prime 4}$ in a Taylor series about $T_{\infty}^{\prime}$ and neglecting the higher order terms, thus, we get

$T^{\prime 4} \cong 4 T_{\infty}^{\prime 3} T^{\prime}-3 T_{\infty}^{\prime 4}$

From Esq. (5) and (6), Eq. (2) reduces to

$\rho c_{p} \frac{\partial T^{\prime}}{\partial t^{\prime}}=k \frac{\partial^{2} T^{\prime}}{\partial y^{\prime 2}}+16 a^{*} \sigma T_{\infty}^{\prime 3}\left(T_{\infty}^{\prime}-T^{\prime}\right)+\frac{D_{m} K_{T} \rho}{C_{s}} \frac{\partial^{2} C^{\prime}}{\partial y^{\prime 2}}$

On introducing the following non- dimensional quantities

$u=\frac{u^{\prime}}{u_{0}}, t=\frac{t^{\prime} u_{0}^{2}}{v}, a_{0}=\frac{v a_{0}^{\prime}}{u_{0}^{2}} \quad y=\frac{y^{\prime} u_{0}}{v}, \theta=\frac{T^{\prime}-T_{\infty}^{\prime}}{T_{w}^{\prime}-T_{\infty}^{\prime}}, C=\frac{C^{\prime}-C_{\infty}^{\prime}}{C_{w}^{\prime}-C_{\infty}^{\prime}}$,

$G r=\frac{g \beta v\left(T_{w}^{\prime}-T_{\infty}^{\prime}\right)}{u_{0}^{3}}, G m=\frac{g \beta^{*} v\left(C_{w}^{\prime}-C_{\infty}^{\prime}\right)}{u_{0}^{3}}, \operatorname{Pr}=\frac{\mu C_{\rho}}{k}, S c=\frac{v}{D}$,

$M=\frac{\sigma B_{0}^{2} v}{\rho u_{0}^{2}}, R=\frac{16 a^{*} v^{2} \sigma T_{\infty}^{\prime 3}}{k u_{0}^{2}}, k=\frac{v k^{\prime}}{u_{0}^{2}}, D u=\frac{D_{m} K_{T}\left(C_{w}^{\prime}-C_{\infty}^{\prime}\right)}{c_{s} c_{p} v\left(T_{w}^{\prime}-T_{\infty}^{\prime}\right)}$

We get the following governing equations which are dimensionless.

$\frac{\partial u}{\partial t}=\frac{\partial^{2} u}{\partial y^{2}}+G r \theta+G m C-M\left(u-K \exp \left(a_{0} t\right)\right)$

$\frac{\partial \theta}{\partial t}=\frac{1}{\operatorname{Pr}} \frac{\partial^{2} \theta}{\partial y^{2}}-\frac{R}{\operatorname{Pr}} \theta+D u \frac{\partial^{2} C}{\partial y^{2}}$

$\frac{\partial C}{\partial t}=\frac{1}{S c} \frac{\partial^{2} C}{\partial y^{2}}-k C$

The initial and boundary conditions in dimensionless form are as follows:

$t \leq 0: u=0, \quad \theta=0, \quad C=0$ for all $y$

$t>0: u=\exp \left(a_{0} t\right), \quad \theta=t, \quad C=1 \quad$ at $y=0$

$$
u \rightarrow 0, \quad \theta \rightarrow 0, \quad C \rightarrow 0 \quad \text { as } y \rightarrow \infty
$$

The appeared physical parameters are defined in the nomenclature.

\section{METHOD OF SOLUTION}

The dimensionless governing equations from (11) to (13), subject to the boundary conditions (14) are solved by usual Laplace transform technique and the solutions are expressed in terms of exponential and complementary error functions.

$C(y, t)=\frac{1}{2}\left[\begin{array}{l}\exp (y \sqrt{k S c}) \operatorname{erfc}\left(\frac{y \sqrt{S c}}{2 \sqrt{t}}+\sqrt{k t}\right) \\ +\exp (-y \sqrt{k S c}) \operatorname{erfc}\left(\frac{y \sqrt{S c}}{2 \sqrt{t}}-\sqrt{k t}\right)\end{array}\right]$

$\theta(y, t)=\left[\begin{array}{l}\left(\frac{t}{2}+\frac{y \operatorname{Pr}}{4 \sqrt{R}}\right) \exp (y \sqrt{R}) \operatorname{erfc}\left(\frac{y \sqrt{\operatorname{Pr}}}{2 \sqrt{t}}+\sqrt{\frac{R t}{\operatorname{Pr}}}\right) \\ +\left(\frac{t}{2}-\frac{y \operatorname{Pr}}{4 \sqrt{R}}\right) \exp (-y \sqrt{R}) \operatorname{erfc}\left(\frac{y \sqrt{\operatorname{Pr}}}{2 \sqrt{t}}-\sqrt{\frac{R t}{\operatorname{Pr}}}\right)\end{array}\right]$

$-\frac{a_{4}}{2}\left[\exp (y \sqrt{R}) \operatorname{erfc}\left(\frac{y \sqrt{\mathrm{Pr}}}{2 \sqrt{t}}+\sqrt{R t}\right)+\exp (-y \sqrt{R}) \operatorname{erfc}\left(\frac{y \sqrt{\mathrm{Pr}}}{2 \sqrt{t}}-\sqrt{R t}\right)\right]$ $+\left(\frac{a_{4}}{2}-\frac{a_{3}}{2}\right) \exp \left(-a_{2} t\right)\left[\begin{array}{l}\exp \left(y \sqrt{R-a_{2} \operatorname{Pr}}\right) \operatorname{erfc}\left(\frac{y \sqrt{\operatorname{Pr}}}{2 \sqrt{t}}+\sqrt{-a_{2} t+\frac{R t}{\operatorname{Pr}}}\right) \\ +\exp \left(-y \sqrt{R-a_{2} \operatorname{Pr}}\right) \operatorname{erfc}\left(\frac{y \sqrt{\operatorname{Pr}}}{2 \sqrt{t}}-\sqrt{-a_{2} t+\frac{R t}{\operatorname{Pr}}}\right)\end{array}\right]$

$+\frac{a_{4}}{2}\left[\exp (y \sqrt{k S c}) \operatorname{erfc}\left(\frac{y \sqrt{S c}}{2 \sqrt{t}}+\sqrt{k t}\right)+\exp (-y \sqrt{k S c}) \operatorname{erfc}\left(\frac{y \sqrt{S c}}{2 \sqrt{t}}-\sqrt{k t}\right)\right]$

$+\left(\frac{-a_{4}}{2}+\frac{a_{3}}{2}\right) \exp \left(-a_{2} t\right)\left[\begin{array}{l}\exp \left(y \sqrt{\left(k-a_{2}\right)} S c\right) \operatorname{erfc}\left(\frac{y \sqrt{S c}}{2 \sqrt{t}}+\sqrt{\left(k-a_{2}\right) t}\right) \\ +\exp \left(-y \sqrt{\left(k-a_{2}\right)} S c\right) \operatorname{erfc}\left(\frac{y \sqrt{S c}}{2 \sqrt{t}}-\sqrt{\left(k-a_{2}\right)}\right)\end{array}\right]$

$u(y, t)=\frac{A_{0}}{2} \exp \left(a_{0} t\right)\left[\begin{array}{l}\exp \left(y \sqrt{M+a_{0}}\right) \operatorname{erfc}\left(\frac{y}{2 \sqrt{t}}+\sqrt{\left(M+a_{0}\right) t}\right) \\ +\exp \left(-y \sqrt{M+a_{0}}\right) \operatorname{erfc}\left(\frac{y}{2 \sqrt{t}}-\sqrt{\left(M+a_{0}\right)} t\right)\end{array}\right]$

$+\frac{A_{1}}{2}\left[\exp (y \sqrt{M}) \operatorname{erfc}\left(\frac{y}{2 \sqrt{t}}+\sqrt{M t}\right)+\exp (-y \sqrt{M}) \operatorname{erfc}\left(\frac{y}{2 \sqrt{t}}-\sqrt{M t}\right)\right]$

$+A_{2}\left[\begin{array}{l}\left(\frac{t}{2}+\frac{y}{4 \sqrt{M}}\right) \exp (y \sqrt{M}) \operatorname{erfc}\left(\frac{y}{2 \sqrt{t}}+\sqrt{M t}\right) \\ +\left(\frac{t}{2}-\frac{y}{4 \sqrt{M}}\right) \exp (-y \sqrt{M}) \operatorname{erfc}\left(\frac{y}{2 \sqrt{t}}-\sqrt{M t}\right)\end{array}\right]$

$+\frac{A_{3}}{2} \exp \left(-a_{6} t\right)\left[\begin{array}{l}\exp \left(y \sqrt{M-a_{6}}\right) \operatorname{erfc}\left(\frac{y}{2 \sqrt{t}}+\sqrt{\left(M-a_{6}\right)} t\right) \\ +\exp \left(-y \sqrt{M-a_{6}}\right) \operatorname{erfc}\left(\frac{y}{2 \sqrt{t}}-\sqrt{\left(M-a_{6}\right)} t\right)\end{array}\right]$

$+\frac{A_{4}}{2} \exp \left(-a_{2} t\right)\left[\begin{array}{l}\exp \left(y \sqrt{M-a_{2}}\right) \operatorname{erfc}\left(\frac{y}{2 \sqrt{t}}+\sqrt{\left(M-a_{2}\right)} t\right) \\ +\exp \left(-y \sqrt{M-a_{2}}\right) \operatorname{erfc}\left(\frac{y}{2 \sqrt{t}}-\sqrt{\left(M-a_{2}\right)} t\right)\end{array}\right]$

$+\frac{A_{5}}{2} \exp \left(-a_{8} t\right)\left[\begin{array}{l}\exp \left(y \sqrt{M-a_{8}}\right) \operatorname{erfc}\left(\frac{y}{2 \sqrt{t}}+\sqrt{\left(M-a_{8}\right)} t\right) \\ +\exp \left(-y \sqrt{M-a_{8}}\right) \operatorname{erfc}\left(\frac{y}{2 \sqrt{t}}-\sqrt{\left(M-a_{8}\right)}\right)\end{array}\right]$

$+A_{6} \exp (-M t) \operatorname{erfc}\left(\frac{y}{2 \sqrt{t}}\right)$

$+\frac{A_{7}}{2}\left[\exp (y \sqrt{R}) \operatorname{erfc}\left(\frac{y \sqrt{\mathrm{Pr}}}{2 \sqrt{t}}+\sqrt{\frac{R t}{\operatorname{Pr}}}\right)+\exp (-y \sqrt{R}) \operatorname{erfc}\left(\frac{y \sqrt{\mathrm{Pr}}}{2 \sqrt{t}}-\sqrt{\frac{R t}{\operatorname{Pr}}}\right)\right]$

$-A_{2}\left[\begin{array}{l}\left(\frac{t}{2}+\frac{y \operatorname{Pr}}{4 \sqrt{R}}\right) \exp (y \sqrt{R}) \operatorname{erfc}\left(\frac{y \sqrt{\operatorname{Pr}}}{2 \sqrt{t}}+\sqrt{\frac{R t}{\operatorname{Pr}}}\right) \\ +\left(\frac{t}{2}-\frac{y \operatorname{Pr}}{4 \sqrt{R}}\right) \exp (-y \sqrt{R}) \operatorname{erfc}\left(\frac{y \sqrt{\operatorname{Pr}}}{2 \sqrt{t}}-\sqrt{\frac{R t}{\operatorname{Pr}}}\right)\end{array}\right]$

$+\frac{A_{8}}{2} \exp \left(-a_{6} t\right)\left[\begin{array}{l}\exp \left(y \sqrt{R-a_{6}} \operatorname{Pr}\right) \operatorname{erfc}\left(\frac{y \sqrt{\operatorname{Pr}}}{2 \sqrt{t}}+\sqrt{-a_{6} t+\frac{R t}{\operatorname{Pr}}}\right) \\ +\exp \left(-y \sqrt{R-a_{6}} \operatorname{Pr}\right) \operatorname{erfc}\left(\frac{y \sqrt{\operatorname{Pr}}}{2 \sqrt{t}}-\sqrt{-a_{6} t+\frac{R t}{\operatorname{Pr}}}\right)\end{array}\right]$

$+\frac{A_{9}}{2} \exp \left(-a_{2} t\right)\left[\begin{array}{l}\exp \left(y \sqrt{R-a_{2}} \operatorname{Pr}\right) \operatorname{erfc}\left(\frac{y \sqrt{\operatorname{Pr}}}{2 \sqrt{t}}+\sqrt{-a_{2} t+\frac{R t}{\operatorname{Pr}}}\right) \\ +\exp \left(-y \sqrt{R-a_{2}} \operatorname{Pr}\right) \operatorname{erfc}\left(\frac{y \sqrt{\operatorname{Pr}}}{2 \sqrt{t}}-\sqrt{-a_{2} t+\frac{R t}{\operatorname{Pr}}}\right)\end{array}\right]$ 


$$
\begin{aligned}
& +\frac{A_{10}}{2}\left[\exp (y \sqrt{k S c}) \operatorname{erfc}\left(\frac{y \sqrt{S c}}{2 \sqrt{t}}+\sqrt{k t}\right)+\exp (-y \sqrt{k S c}) \operatorname{erfc}\left(\frac{y \sqrt{S c}}{2 \sqrt{t}}-\sqrt{k t}\right)\right] \\
& +\frac{A_{11}}{2} \exp \left(-a_{2} t\right)\left[\begin{array}{l}
\exp \left(y \sqrt{\left(k-a_{2}\right) S c}\right) \operatorname{erfc}\left(\frac{y \sqrt{S c}}{2 \sqrt{t}}+\sqrt{\left(k-a_{2}\right) t}\right) \\
+\exp \left(-y \sqrt{\left(k-a_{2}\right) S c}\right) \operatorname{erfc}\left(\frac{y \sqrt{S c}}{2 \sqrt{t}}-\sqrt{\left(k-a_{2}\right) t}\right)
\end{array}\right] \\
& +\frac{A_{12}}{2} \exp \left(-a_{8} t\right)\left[\begin{array}{l}
\exp \left(y \sqrt{\left(k-a_{8}\right) S c}\right) \operatorname{erfc}\left(\frac{y \sqrt{S c}}{2 \sqrt{t}}+\sqrt{\left(k-a_{8}\right) t}\right) \\
+\exp \left(-y \sqrt{\left(k-a_{8}\right) S c}\right) \operatorname{erfc}\left(\frac{y \sqrt{S c}}{2 \sqrt{t}}-\sqrt{\left(k-a_{8}\right) t}\right)
\end{array}\right] \\
& +A_{6} \exp \left(a_{0} t\right)-A_{6} \exp (-M t)
\end{aligned}
$$

\subsection{Nusselt Number}

From temperature field, the Nusselt number which is given in nondimensional form as follows:

$N u=-\left[\frac{\partial \theta}{\partial y}\right]_{y=0}$

From equations (16) and (18), we get Nusselt number as follows

$$
\begin{aligned}
& N u=-\left[t \sqrt{R} \operatorname{erf} \sqrt{\frac{R t}{\operatorname{Pr}}}-\sqrt{\frac{t \operatorname{Pr}}{\pi}} \exp \left(-\frac{R t}{\operatorname{Pr}}\right)-\frac{\operatorname{Pr}}{2 \sqrt{R}} \operatorname{erf} \sqrt{\frac{R t}{\operatorname{Pr}}}\right] \\
& -a_{4}\left[\left(-\exp \left(-\frac{R t}{\operatorname{Pr}}\right)\right) \sqrt{\frac{\operatorname{Pr}}{\pi t}}-\sqrt{R} \operatorname{erf}\left(\sqrt{\frac{R t}{\operatorname{Pr}}}\right)\right] \\
& -\left(a_{4}-a_{3}\right) \exp \left(-a_{2} t\right)\left[\left(-\exp \left(-\frac{R t}{\operatorname{Pr}}+a_{2} t\right)\right) \sqrt{\frac{\operatorname{Pr}}{\pi t}}-\sqrt{R-a_{2} \operatorname{Pr}} \operatorname{erf}\left(\sqrt{\frac{R t}{\operatorname{Pr}}-a_{2} t}\right)\right] \\
& +a_{4}\left[\sqrt{\frac{S c}{\pi t}} \exp (-k t)+\sqrt{k S c} \operatorname{erf} \sqrt{k t}\right] \\
& -\left(a_{3}-a_{4}\right) \exp \left(-a_{2} t\right)\left[\left(-\exp \left(-k t+a_{2} t\right)\right) \sqrt{\frac{S c}{\pi t}}-\sqrt{k S c-a_{2} S c} \operatorname{erf}\left(\sqrt{k t-a_{2} t}\right)\right]
\end{aligned}
$$

\subsection{Sherwood Number}

From concentration field, Sherwood number which is given in non dimensional form as follows:

$S h=-\left[\frac{\partial C}{\partial y}\right]_{y=0}$

From equations (15) and (20) we get Sherwood number as follows:

$$
S h=\left[\exp (-k t) \sqrt{\frac{S c}{\pi t}}+\sqrt{k S c} \operatorname{erf}(\sqrt{k t})\right]
$$

\section{RESULTS AND DISCUSSION}

In order to get a clear insight of the physical problem, the velocity, temperature, concentration, the rate of heat transfer and the rate of mass transfer have been discussed by assigning numerical values to the parameters like radiation parameter $(R)$, magnetic parameter $(M)$,exponential accelerated parameter $\left(a_{0}\right)$, Schmidt parameter $(S c)$, Prandtl number $(P r)$, Dufour number $(D u)$, thermal Grashof number $(G r)$, mass Grashof number $(G m)$, chemical reaction $(k)$ and time $(t)$ from Figs. 2 -14.

Concentration profiles for different values of Schmidt number $S c$ and chemical reaction parameter kare presented through Fig. 2reveals that the concentration field due to variation in Schmidt number for the gasses hydrogen $(S c=0.22)$, water vapour $(S c=0.60)$, ammonia $(S c=0.78)$, carbon dioxide $(S c=0.96)$. It is observed that concentration field is arrived regularly for hydrogen and accrues for carbon dioxide in comparison to watervapour. Thus, watervapour can be used for maintaining concentration field and hydrogen can be used for maintaining good concentration field. An increasing in Schmidt number leads to decreases in the concentration boundary layer thickness. From Fig. 3it is seen that the concentration decreases with an increasing value of $k$.

The influence of various flow parameters on the fluid temperature are illustrated in Figs. 4 -7. Fig. 4 depicts that the effects of the Dufour number on the fluid temperature. It can be clearly seen from this figure that the diffusion thermal effects show the significant effect on the fluid temperature. As the values of Dufour number increases, the fluid temperature is also enhanced. The various values of Prandtl number are chosen such that for air $(\operatorname{Pr}=0.71)$, electrolytic solution $(\operatorname{Pr}=1.0)$ and water $(P r=7.0)$. Fig.5revealsthat the temperature decreases with the increasing values of Prandtl number. This is due to the fact that an increment in Prandtl number fluid has comparatively low thermal conductivity, which reduces conduction and there by the thermal boundary layer thickness and as a result, temperature decreases. Effect of thermal radiation $R$ on the temperature field is illustrated in Fig. 6 illustrates that the radiation parameter restricts the fluid temperature. Therefore, using radiation we can control the fluid temperature. The temperature profiles at different time are shown in Fig. 7 it is observed that the temperature is increasing with time and approach to a steady state temperature as the time arises. The velocity profiles for an exponentially accelerated plate are presented from Figs. $8-13$ when the magnetic lines of force are fixed relative to the fluid $(K=0)$ or to the plate $(K=1)$. The effects of $M, D u, G r, G m$, an and $t$ on the velocity field are shown in Figs. $8-13$ in the presence of radiation and chemical reaction respectively when other parameters fixed.

Fig.8 depicts that the velocity various magnetic parameter. It is observed that an increase in magnetic parameter the velocity decreases. It is due to the fact that the application of transverse magnetic field will result a Lorentz force similar to drag force, which tends to resist the fluid flow and thus reducing its velocity and it is also noticed that the momentum boundary layer thickness increases with increasing value of magnetic parameter in case of moving plate. From these Fig. 9 it is noticed that the velocity increases with the increasing values of $D u$. It can be clearly seen from this figure that the diffusion thermal effects show the significant effect on the fluid velocity. The effects of $G r, G m$ and $t$ on the velocity field are shown in figures $10-12$. It is observed that the velocity of the fluid increases with increasing values of $\mathrm{Gr}, \mathrm{Gm}$ and $t$. This is because an increase in $G r$ or $G m$ or $t$ leads to an increase in the buoyancy force which causes an increase in the fluid velocity. From Fig. 13reveals that the velocity increases with in increasing exponential accelerated parameter. 


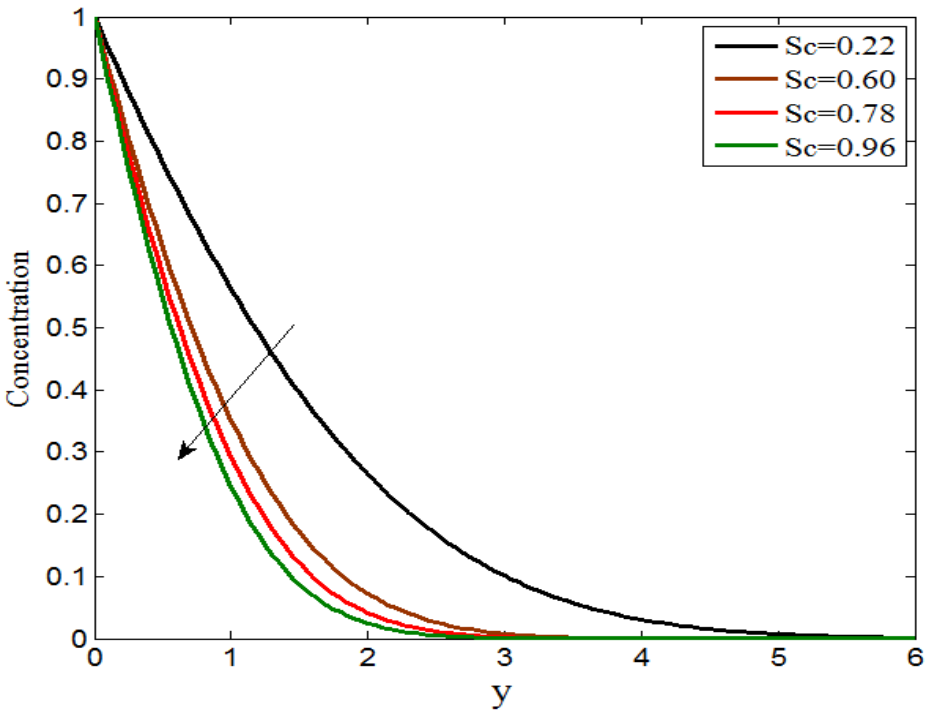

Fig. 2 Concentration profiles for various values of $S c$ when $k=0.5$, $t=0.4$

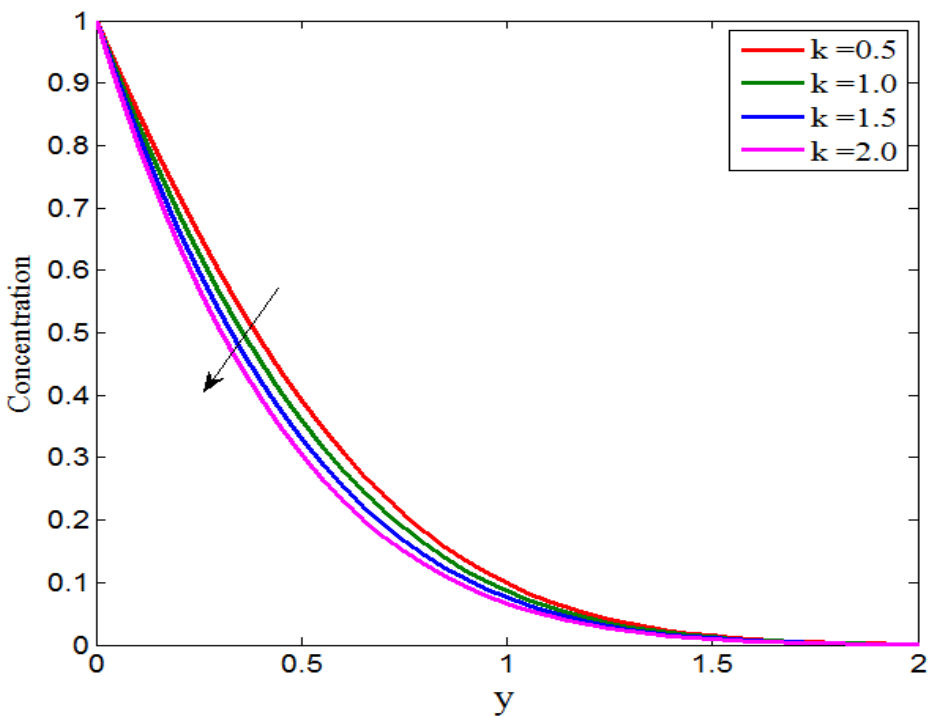

Fig. 3 Concentration profiles for various values of $k$ when $S c=2.01$, $t=0.4$

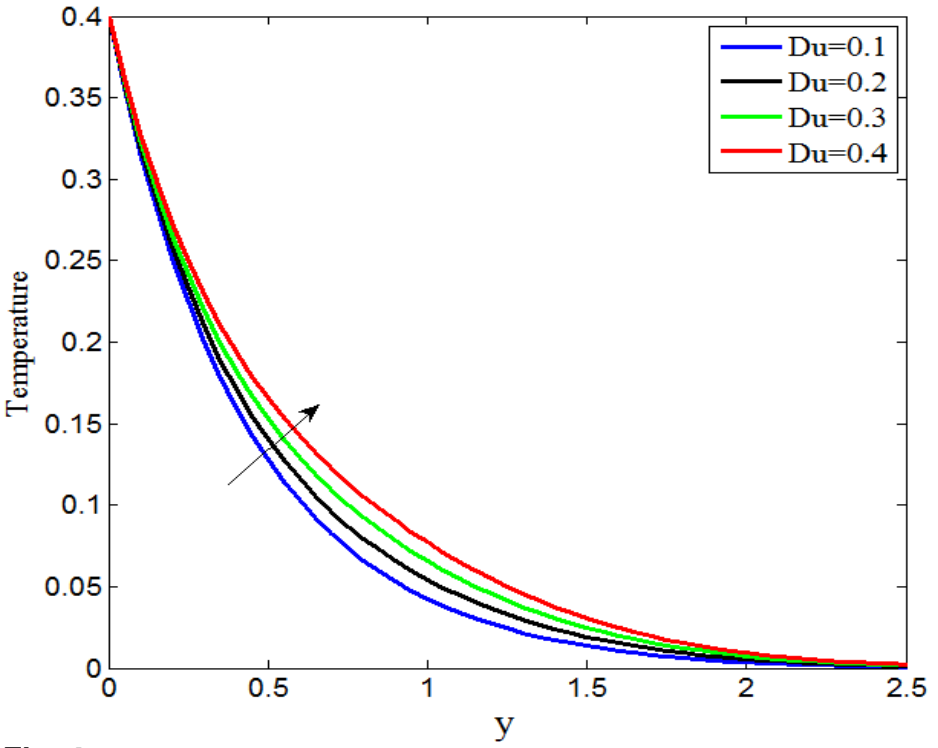

Fig. 4 Temperature profiles for various values of $D u$ when $S c=2.01$, $t=0.4, \operatorname{Pr}=0.71, R=4, k=0.5$

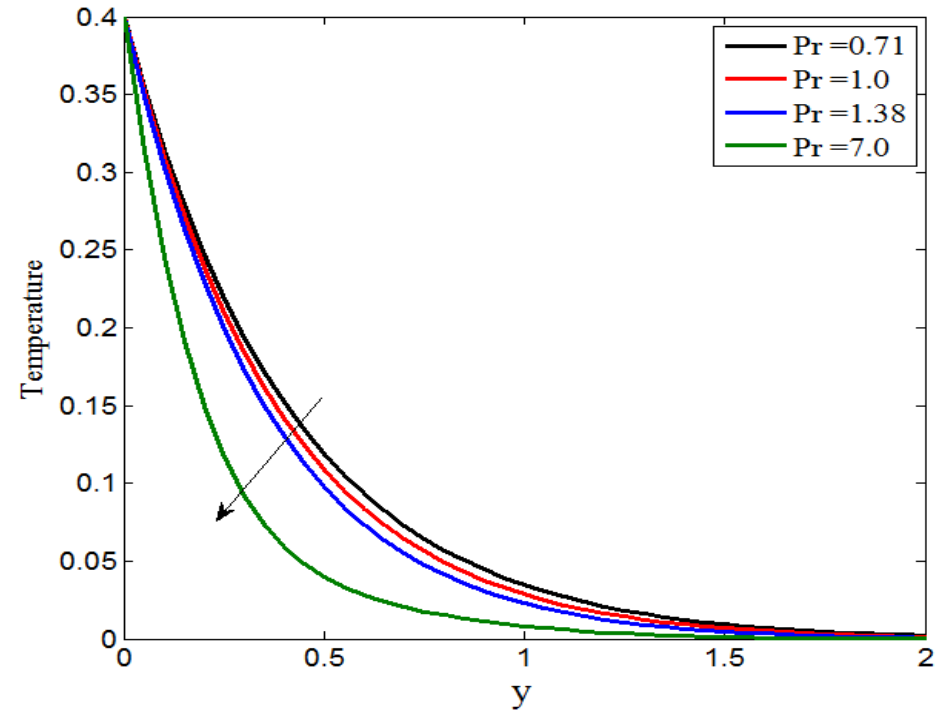

Fig. 5 Temperature profiles for various values of $\operatorname{Pr}$ when $S c=2.01$, $t=0.4, D u=0.03, R=4, k=0.5$

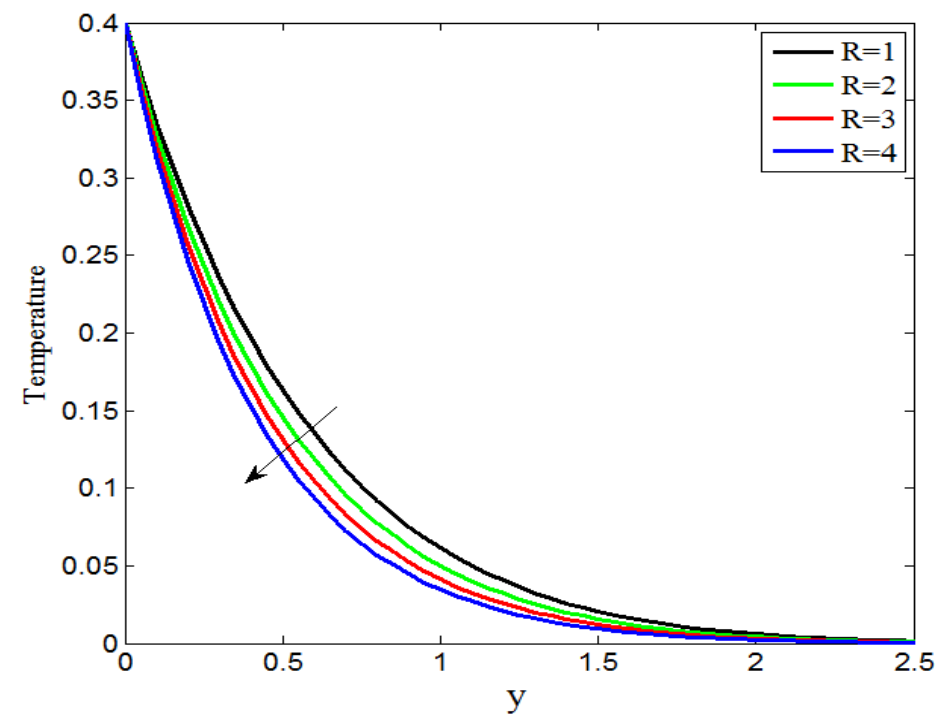

Fig. 6 Temperature profiles for various values of $R$ when $S c=2.01$, $t=0.4, D u=0.03, P r=0.71, k=0.5$

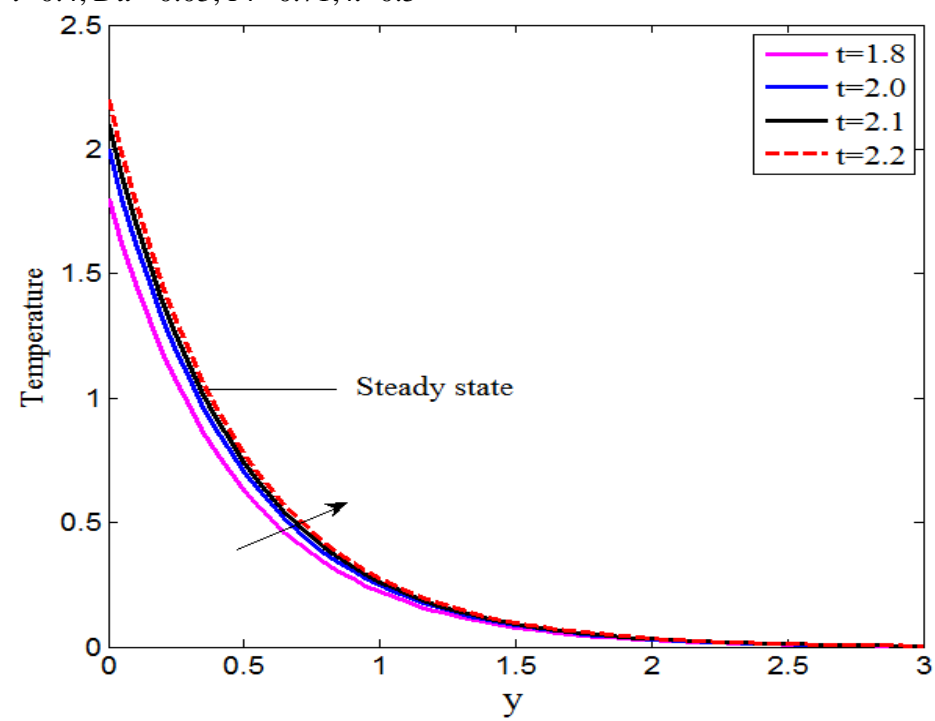

Fig. 7 Temperature profiles for various values of $t$ when $S c=2.01, D u$ $=0.03, \operatorname{Pr}=0.71, R=4, k=0.5$ 


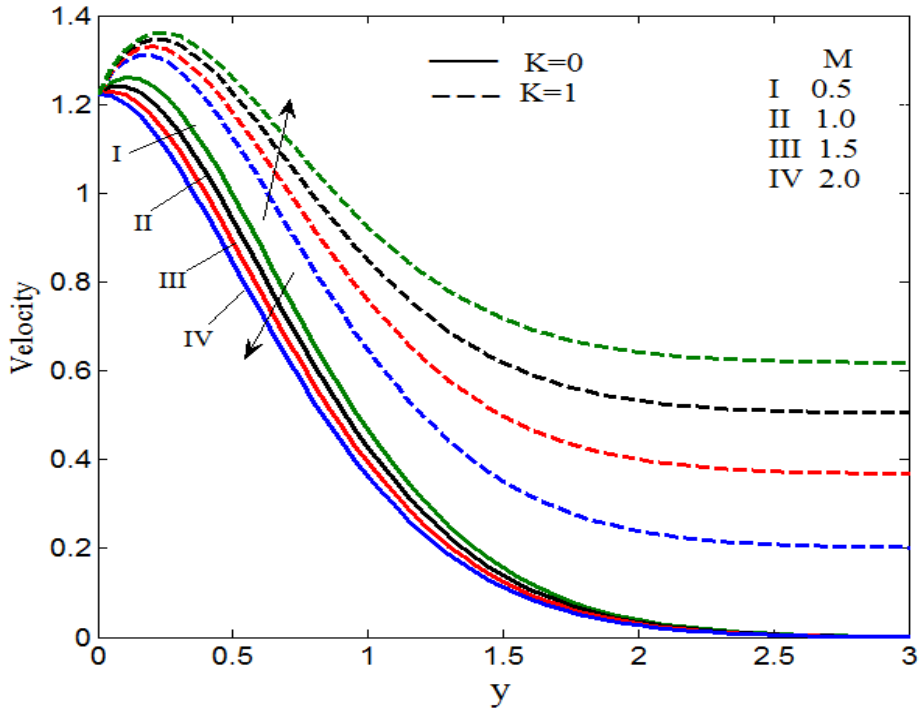

Fig. 8 Velocity profiles for various values of $M$ when $S c=2.01, D u$ $=0.03, G r=10, G m=5 a_{0}=0.5, P r=0.71, R=4, k=0.5, t=0.4$

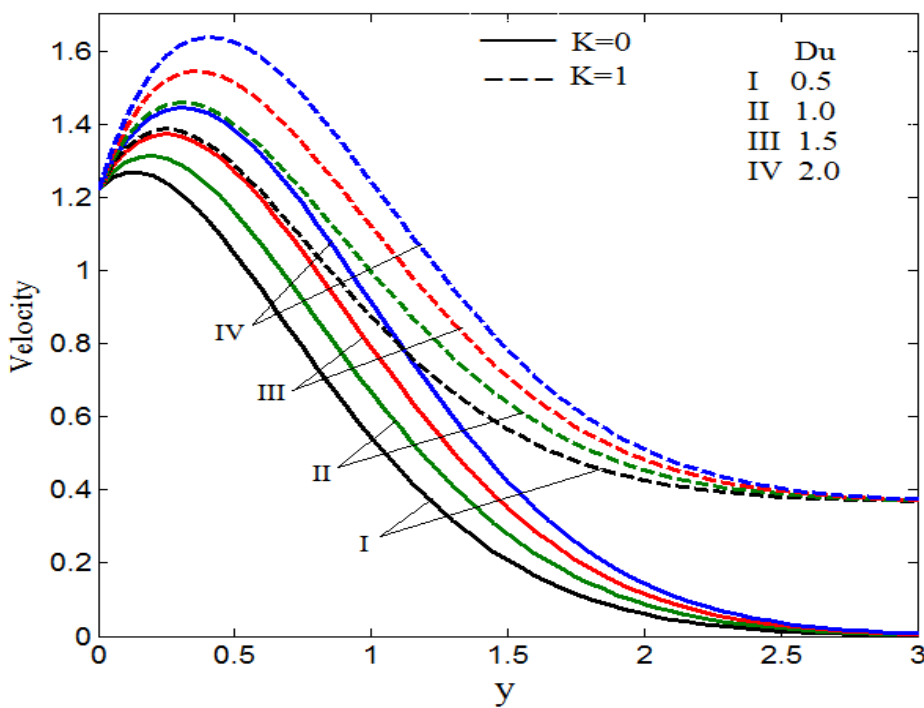

Fig. 9 Velocity profiles for various values of $D u$ when $S c=2.01, M=1$, $G r=10, G m=5, a_{0}=0.5, \operatorname{Pr}=0.71, R=4, k=0.5, t=0.4$

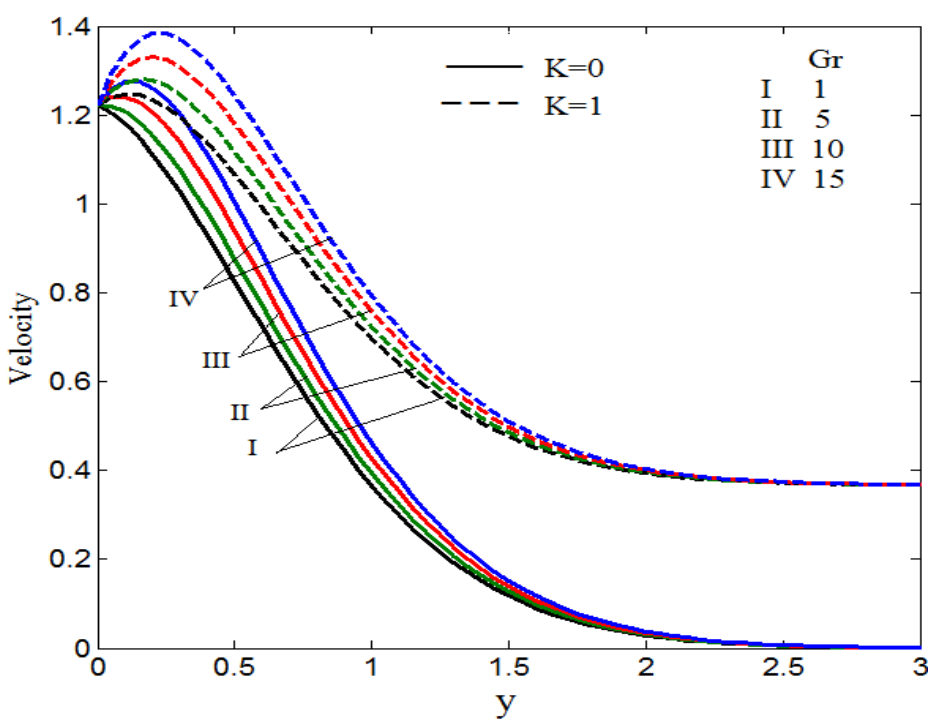

Fig. 10 Velocity profiles for various values of $G r$ when $S c=2.01, D u$ $=0.03, M=1, G m=5 a_{0}=0.5, P r=0.71, R=4, k=0.5, t=0.4$

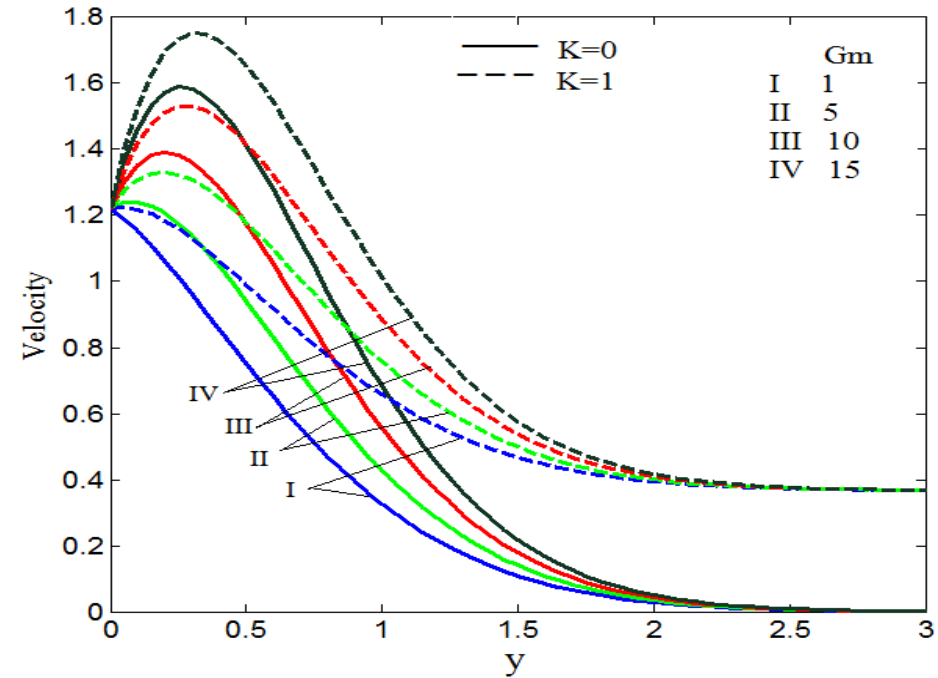

Fig. 11 Velocity profiles for various values of $G m$ when $S c=2.01, D u$ $=0.03, G r=10, M=1 a_{0}=0.5, P r=0.71, R=4, k=0.5, t=0.4$

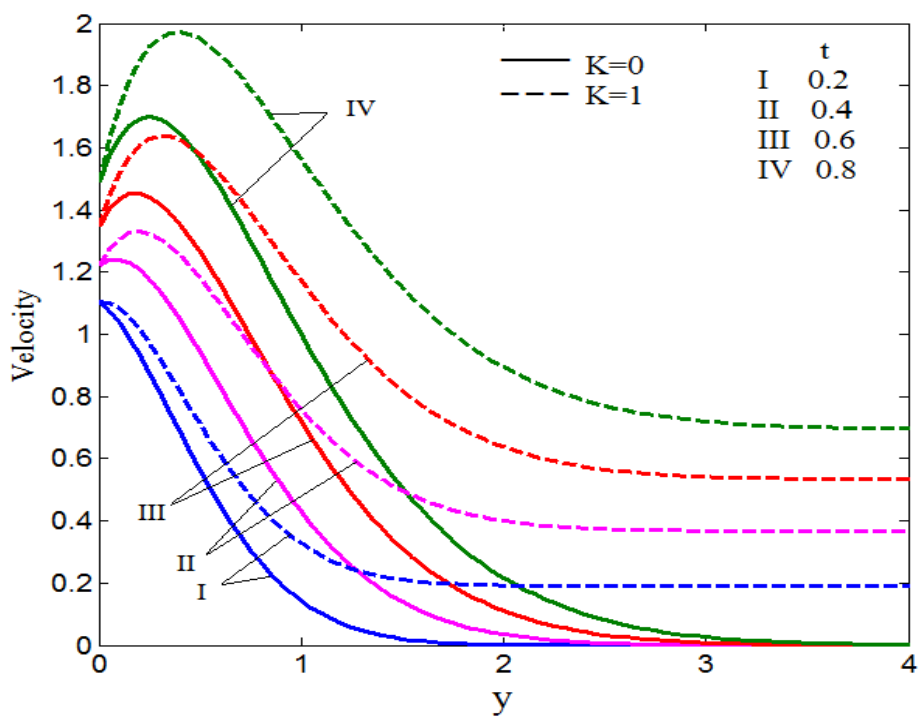

Fig. 12 Velocity profiles for various values of $t$ when $S c=2.01, D u$ $=0.03, G r=10, G m=5 a_{0}=0.5, P r=0.71, R=4, k=0.5, M=1$

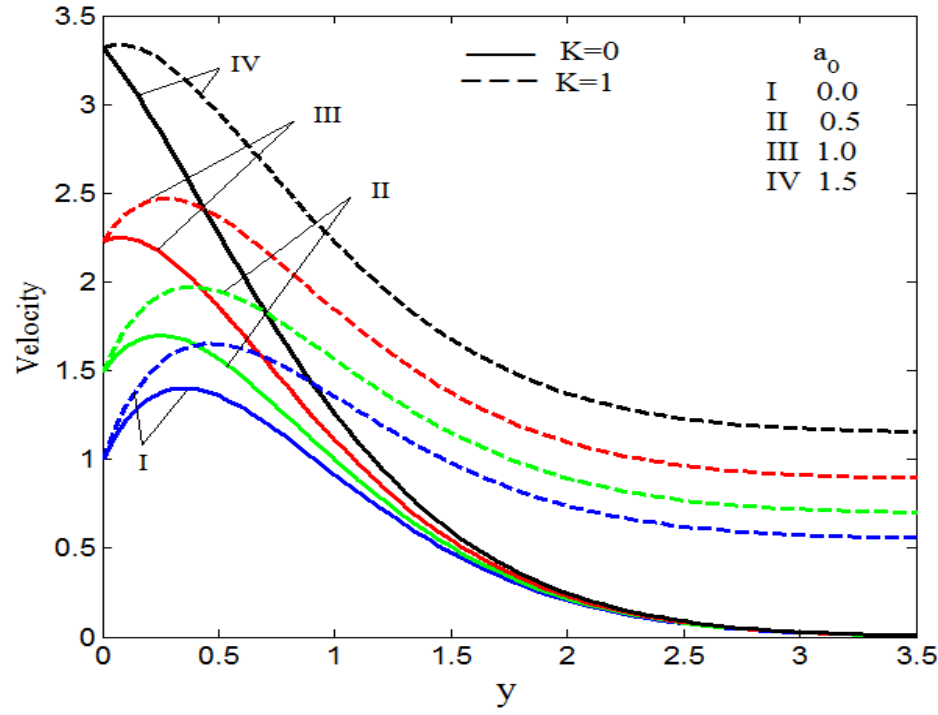

Fig. 13 Velocity profiles for various values of $a_{0}$ when $S c=2.01, D u$ $=0.03, G r=10, G m=5, P r=0.71, R=4, k=0.5, M=1, t=0.4$ 


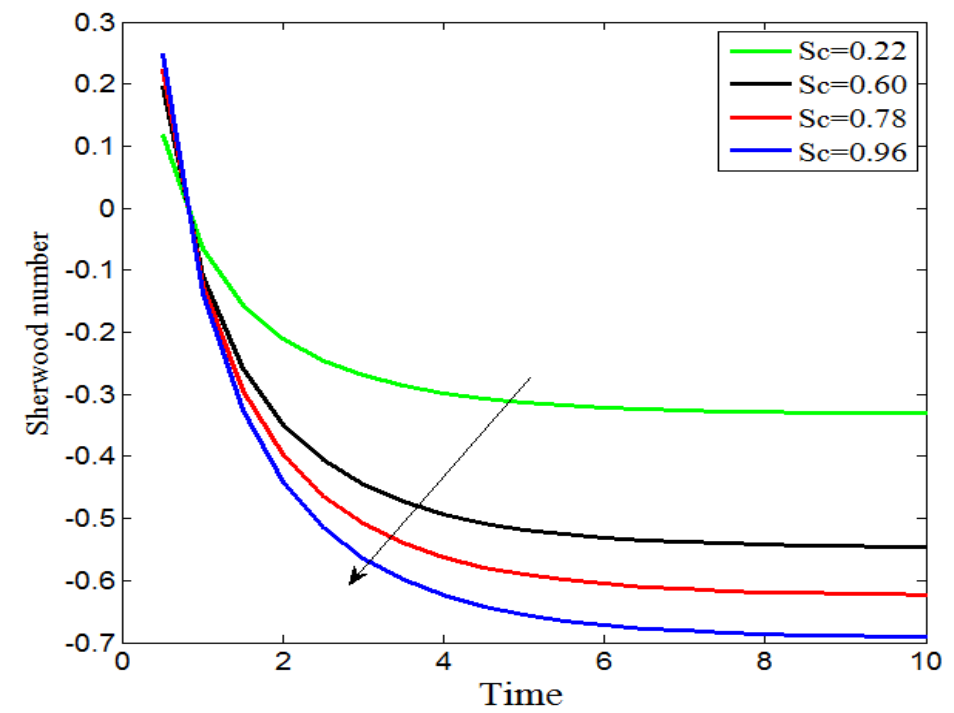

Fig. 14 Sherwood number for various values of $S c$ when $k=0.5$

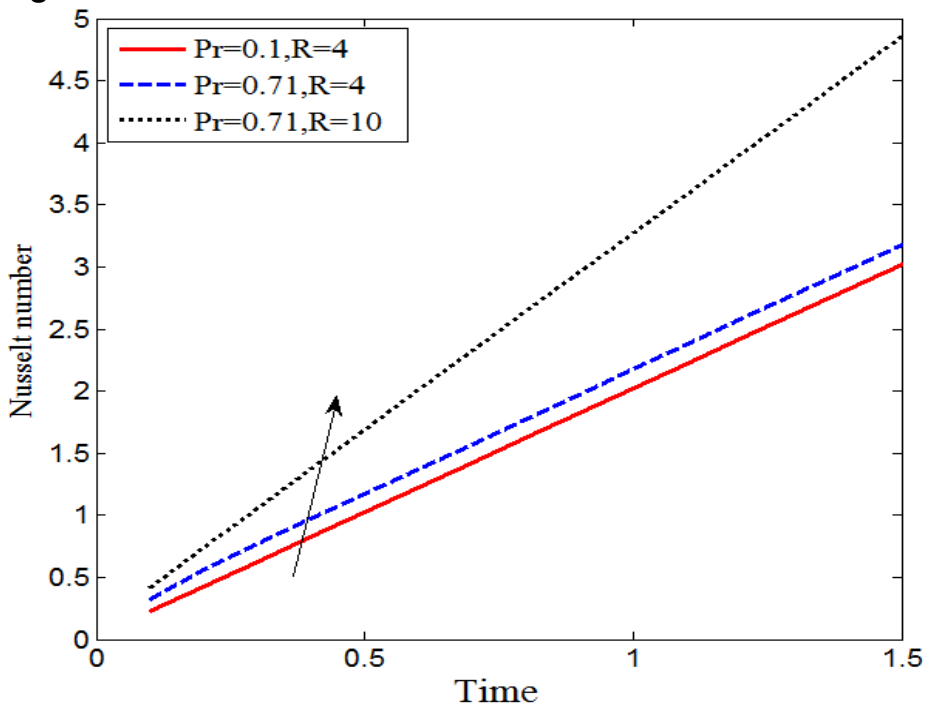

Fig. 15 Nusselt number for various values of $\operatorname{Pr}$ and $R$ when $S c=2.01$, $k=0.5, D u=0.03$

Finally, the rate at which mass transfers and the rate at mass transfers are examined through the figures 14 and 15 respectively. From Fig.14 it is seen that for the fixed values of $k$ the Sherwood number $S h$ increases as $S c$ decreases. Figure 15 reveals that the rate of heat transfer coefficient $N u$ for different values of Prandtl number $P r$, radiation parameter $R$ and Dufour number $D u$ against time $t$. It is observed that for fixed values of $D u$ the Nusselt number increases with increasing values of $\operatorname{Pr}$ or $R$.

\section{CONCLUSIONS}

A mathematical model has been presented fordiffusion-thermo and radiation effects on unsteady MHD free convection of flow of a viscous, incompressible, electrically conducting fluid past an accelerated infinite plate with variable temperature and uniform mass diffusion in the presence of transverse applied magnetic field.Laplace transform solutions for the non-dimensional momentum, energy and concentration equations subject to boundary conditions have been obtained. The expressions for Nusselt number and Sherwood number at the plate are obtained and solutions are presented graphically for the pertinent governing parameters.From this investigation, the fallowing conclusions arrive.
- The concentration profiles decrease with the increase of $S c$ and $k$.

- $\quad$ The temperature profiles increase with the increasing values of $D u$ or $P r$ but reverse trend is obtained for $R$

- $\quad$ The velocity of the fluid decreases with an increasing values of $M$

- The velocity of the fluid increases with an increasing valves of $\mathrm{Du}$ or $\mathrm{Gr}$ or $\mathrm{Gm}$

- In general, the flow field, temperature, and concentration distribution are affected by the physical parameters.

\section{NOMENCLATURE}

$a_{0}$ Accelerated parameter

$B_{0}$ External magnetic field (A.m $\left.{ }^{2}\right)$

$C^{\prime}$ Species concentration $\left(\mathrm{kg} \mathrm{m}^{-3}\right)$

$C_{w}^{\prime}$ Concentration of the plate $\left(\mathrm{kg} \mathrm{m}^{-3}\right)$

$C_{\infty}^{\prime}$ Concentration of the fluid far away from the plate $\left(\mathrm{kg} \mathrm{m}^{-3}\right)$

$C$ Dimensionless concentration $\left(\mathrm{kg} \mathrm{m}^{3}\right)$

$C_{p}$ Specific heat at constant pressure $\left(\mathrm{J} \mathrm{kg}^{-1} \mathrm{~K}\right)$

$D u \quad$ Dufour effect

$g$ Acceleration due to gravity $\left(\mathrm{ms}^{-2}\right)$

$G r$ Thermal Grashof number

Gm Mass Grashof number

$M \quad$ Magnetic field parameter $\left(\mathrm{Am}^{-1}\right)$

$N u$ Nusselt number

Pr Prandtl number

$q_{r} \quad$ Radiative heat fluxes in the y-direction

$D_{m}$ Coefficient of mass diffusivity

$R$ Radiation parameter $\left(\mathrm{cm}^{2}\right)$

$S c$ Schmidt number

$T^{\prime}$ Temperature of the fluid near the plate $(\mathrm{K})$

$T_{w}^{\prime}$ Temperature of the plate (K)

$T_{\infty}^{\prime}$ Temperature of the fluid far away from the plate (K)

$t^{\prime} \quad$ Time

$t$ Dimensionless time (Sec)

$u^{\prime}$ Velocity of the fluid in the $y^{\prime}$-direction

$u_{0}$ Velocity of the plate

$u$ Dimensionless velocity $\left(\mathrm{ms}^{-1}\right)$

$y^{\prime}$ Coordinate axis normal to the plate

$y$ Dimensionless Coordinate axis normal to the plate

\section{Greek symbols}

$k$ Thermal conductivity of the fluid $\left(\mathrm{Wm}^{-1} \mathrm{~K}^{-1}\right)$

$\alpha$ Thermal diffusivity $\left(\mathrm{Wm}^{2} \mathrm{j}^{-1}\right)$

$\beta$ Volumetric coefficient of thermal expansion $\left(\mathrm{K}^{-1}\right)$

$\beta^{*}$ Volumetric coefficient of expansion with concentration

$\mu$ Coefficient of viscosity $\left(\mathrm{m}^{2} \mathrm{~s}^{-1}\right)$

$v$ Kinematic viscosity $\left(\mathrm{m}^{2} \mathrm{~s}^{-1}\right)$

$\rho$ Density of the fluid $\left(\mathrm{kg} \mathrm{m}^{-3}\right)$

$\sigma \quad$ Electric conductivity $\left(\mathrm{Sm}^{-1}\right)$

$\theta \quad$ Dimensionless temperature (K)

\section{Subscripts}

$w$ Conditions on the wall

$\infty$ Freestream conditions 


\section{ACKNOWLEDGEMENTS}

The authors are very thankful to the reviewer and editor for their suggestions, which have significantly improved the quality of our research paper.

\section{REFERENCES}

Abramowitz, M., Stegu,A.I.:Handbook of Mathematical Functions, Sect. 7.4.33 (Dover Publications, New York, 1972).

Alam, M. S., Rahman, M. M., 2005, "Dufour and Soret effects on MHD Free Convection Heat and Mass Transfer Flow past a Vertical Flat Plate embedded in a Porous Medium". Journal of Naval Architecture and Marine Engineering. 2(1), 55-65.

Alam, M.S., Rahman, M. M., 2006, "Dufour and Soret effects on Mixed Convection Flow past a Vertical Porous Flat Plate Embedded in a Porous Medium with Variable Suction". Journal of Nonlinear Analysis:Model.Control. 11(1),3-12.

Alam, M. S., Rahman, M.M., Smad, M.A., 2006a, "Dufour and Soret Effects on Unsteady MHD Free Convection and Mass Transfer Flow past a Vertical Porous Plate in a Porous Medium". NonlinearModel.Control. 11(3), 217-226.

Alam, M.S., Ferdows, M., Ota, M., Maleque, M.A., 2006b, “ Dufour and Soret Effects on Steady Free Convection and Mass Transfer Flow past a Semi-Infinite Vertical Porous Plate in a Porous Medium. International Journal of AppliedMechanics and Engineering. 11(3), $535-545$.

Chandran, P., Sacheti,N. C., Singh, A. K. 1998, "Unsteady Hydro magnetic Free Convection Flow with Heat Flux and Accelerated Boundary motion. Journal of the Physical Society of Japan.67, 124129.

http://dx.doi.org/10.1143/jpsj.67.124

Cramer, K. R., Pai, S.I., Magneto Fluid Dynamics for Engineers and Applied Physicists (McGraw-Hill Book Company, NewYork, 1973).

Eckert, E.R.G., Drake, R.M., Analysis of Heat and Mass Transfer. McGraw-Hill, New York (1972).

http://dx.doi.org/10.1002/aic.690180342

Georgantopoulos, G. A., Douskos, C. N., Kafousiasand, N.G., Goudas, C. L., 1979, "Hydromagnetic Free Convection Effects on the Stokes problem for an Infinite Vertical Plate". Letters Heat and Mass Transfer. 6, 397-404.

http://dx.doi.org/10.1016/0094-4548(79)90050-x

Hetnarski, R. B., 1975, "An Algorithm for generating some Inverse Laplace Transforms of Exponential Form" ZeitschriftfürangewandteMathematik und Physik. 26, 249-253. http://dx.doi.org/10.1007/bf01591514

Prakash, J., Bhanumathi, D., Kumar, A. G. V. and Varma, S. V. K., 2013, "Diffusion-Thermo and Radiation Effects on Unsteady MHD Flow through Porous Medium Past an Impulsively Started Infinite Vertical Plate with Variable Temperature and Mass Diffusion," Transport in Porous Media, Springer, 96(1), 135-151.

http://dx.doi.org/10.100/s11242-012-0078-x

Kumar, B.R., Sravan Kumar, T., and Kumar, A G V., 2015.“Thermal Diffusion and Radiation Effects on Unsteady Free Convection Flow In the Presence of Magnetic Field Fixed Relative to the Fluid or the Plate". Frontiers Heat and Mass Transfer, 6,12,.1-9.

http://dx.doi.org/10.5098/hmt.6.12
Muralidharan, M., Muthucumaraswamy, R., 2013, "Radiation Effects on Linearly Accelerated Isothermal Plate with Variable Mass Diffusion in the Presence of Magnetic Field". Applied Mathematical Sciences. 7(113), 5645-5656. http://dx.doi.org/10.12988/ams.2013.38458

Muthucumaraswamy, R.,Sathappan, K.E., Natarajan, R., 2004, "Mass transfer Effects on Exponentially Accelerated Isothermal Vertical Plate". International Journal of AppliedMathematics and Mechics. 4(6), 19-25. https://doi.org/10.2298/tsci1001073m

Mythreye, A., Pramod, J.P., Balamurugan, K. S., 2015, "Chemical Reaction on Unsteady MHD Convective Heat and Mass Transfer past a Semi Infinite Vertical Permeable Moving Plate with Heat Absorption”. Procedia Engineering. 127, 613-620.

https://doi.org/10.1016/j.proeng.2015.11.352

Narahari, M., Debnath, L., 2013, "Unsteady Magneto-hydrodynamic Free Convection Flow Past an Accelerated Vertical Plate with Constant Heat Flux and Heat generation or absorption”. Journal of Applied Mathematics and Mechanics / Zeitschriftfür Angewandte Mathematik und Mechanik. 93(1), 38 - 49.

http://dx.doi.org/10.1002/zamm.201200008

Ogulu, A., Makinde, O. D., 2009, "Unsteady Hydromagnetic Free Convection Flow of a Dissipative and Rotating Fluid past a Vertical Plate with Constant Heat Flux". Chemical Engineering Communication.196, 454-462. https://doi.org/10.1080/00986440802484531

Prabhakar Reddy, B., 2014 "Effects of Thermal Diffusion and Viscous Dissipation on Unsteady MHD Free Convection flow past a Vertical Porous Plate under Oscillatory Suction Velocity with Heat Sink". International Journal of Applied Mechanics and Engineering. 19(2), 303-320.

http://dx.doi.org/10.2478/ijame-2014-0020

Postelnicu, A., 2004, "Influence of a Magnetic Field on Heat and Mass transfer by Natural Convection from Vertical Surfaces in Porous Media considering Soret and Dufour effects”. International Journal of Heat and Mass Transfer.47, 1467-1472.

http://dx.doi.org/10.1016/j.ijheatmasstransfer.2003.09.017

Puri, P., Kythe, P.K., 1998, "Some Inverse Laplace transforms of Exponential form". ZeitschriftfürangewandteMathematik und Physik. 39,150-156.

http://dx.doi.org/10.1007/bf00945134

Raju, R.S., Sudhakar, K., Rangamma, M., 2013, "The Effects of Thermal Radiation and Heat Source on an Unsteady MHD Free Convection flow past an Infinite Vertical Plate with Thermal Diffusion and Diffusion Thermo". The Institution of Engineers (India): Series $C$. 94(2), $175-186$.

http://dx.doi.org/10.1007/s40032-013-0063-3

Rajesh, V., Varma, S.V. K., 2009, "Radiation and Mass Transfer Effects on MHD Free Convection Flow past an Exponentially Accelerated Vertical Plate with Variable Temperature". ARPN Journal of Engineering Applied Sciences. 4(6), 20-26.

Raptis, A., Singh, A.K., 1983, "MHD Free Convection Flow past an Accelerated Vertical Plate". International Communications in Heat and Mass Transfer. 10, 313-321.

https://doi.org/10.1016/0735-1933(83)90016-7 
Seth, G. S., Ansari, Md. S., Nandkeolyar, R., 2010, "MHD Natural Convection Flow past an Impulsively Moving Vertical Plate with Ramped wall Temperature in the Presence of Thermal Diffusion with Heat absorption". International Journal of AppliedMechanics and Engineering. 15, 199-215.

https://doi.org/10.1007/s00231-010-0740-1

Sivaiah, S., Anitha, K., Venkataramana, S., 2012, "Effects of Thermal Diffusion and Radiation on Unsteady MHD Free Convection Flow Past an Infinite Heated Vertical Plate in a Porous Medium". Journal Article published in ISRN Thermodynamics. 2012, 1- 8.

http://dx.doi.org/10.5402/2012/670396

Swetha Ravi., Prakash, J.,Viswanatha Reddy Gottam., Varma, S.V.K., 2015, "Effects of Thermal Radiation and Radiation Absorption on Flow past an Impulsively Started Infinite Vertical Plate with Newtonian Heating and Chemical Reaction". Open journal of Fluid Dynamics. 5(04), 364-379.

https://doi.org/10.4236/ojfd.2015.54036
Venkateswarlu, M., Ramana Reddy, G.V., Lakshmi, D. V., 2014, "Thermal Diffusion and Radiation Effects on Unsteady MHD Free Convection Heat and Mass Transfer Flow Past a Linearly Accelerated Vertical Porous Plate with Variable Temperature and Mass Diffusion". The Korea Society for Industrial and Applied Mathematics. 183, 257 268.

http://dx.doi.org/10.12941/jksiam.2014.18.257

Tokis, J. N., 1985, "A Class of Exact Solutions of the Unsteady Magnetohydrodynamic Free-convection Fows". Astrophysics and Space Science.112, 413-422.

Toki, C. J., Tokis, J. N., 2007, "Exact Solutions for the Unsteady Free Convection Flows on a Porous Plate with Time-dependent Heating". ZeitschriftfürAngewandteMathematik und Mechanik. 87, 4-13. http://dx.doi.org/10.1002/zamm.200510291/. 